\title{
Design of Four-Axis Winding Machine for Tapered Composite Pole Using Heated-Mandrel Curing
}

\author{
Jiazhong $\mathrm{Xu}^{1,2}$, Rongguo Wang ${ }^{1}$, Tao Sun ${ }^{2}$, \\ Qingmin Huang ${ }^{2}$ and Honghong Yang ${ }^{2}$ \\ ${ }^{1}$ Harbin Institute of Technology. 92, West Dazhi Street, \\ Nangang District, Harbin, China \\ ${ }^{2}$ Harbin University of Science and Technology. \\ 52, Xuefu Road, Nangang District, Harbin, China \\ xujiazhong@163.com
}

\begin{abstract}
A four-axis winding machine for tapered pole using heated-mandrel curing method is designed in this paper. The control system of this winding machine is designed based on IPC and embedded motion controller. Electronic gear is used to realize the synchronous motion control of the carriage, the beam, and the winding guide head. The mandrel structure is optimized to realize the uniformity of the temperature distribution in the axial direction based on numerical simulation. Experiments show that the winding pattern of this winding machine is stable, and the curing quality is high.
\end{abstract}

Keywords: composite, tapered pole, winding machine, control system

\section{Introduction}

Reinforced concrete telegraph towers and wooden poles both have defects, such as heavy weight, inconvenience for transport, vulnerability to pests, and perishability under the longterm erosion of the water [1-2]. With the advantages of light weight, high strength, fireproof, heat resistant, ultraviolet resistant, pest resistant, excellent insulation, easy forming and easy installation, the composite power pole is widely used in power transmission field [3]. The use of the composite pole not only decreases the installation cost, but also has considerable economic and social benefits [4].

The composite power pole is mainly applied and manufactured in North America and Europe. The Shakespeare Composite Structures was the first company to develop the composite pole in 1954. The RS, the Ebert and other foreign companies are also involved in the development, industrial manufacture and applied research of the composite pole [5]. Besides, many researches are employed on the production process of FRP poles. Foumier proposed the concept of composite modular poles [6]. Metiche analyzed the influences of the relative location of the opening from the base of the pole to the performance of the FRP poles, and used a new approach to accurately evaluate the design equations for the flexural behavior of FRP poles [7]. Fam analyzed the influences of fiber orientation, thickness, and the diameter of the central hole to the stiffness of concrete-filled FRP tubes used for poles [8]. Masmoudi evaluated the deflection and bending strength of GFRP poles, and optimized the structure of the poles, making its load carrying capacity and flexural stiffness improved with a significant saving in the weight [9]. 
This paper designs an heated-mandrel curing winding machine for tapered composite power pole. The control system is designed, and the synchronized motion control of the carriage, the outrigger, and the winding guide head tracking the spindle is realized by the electronic gear.

\section{Control Principle Analysis and Winding Pattern Design}

\subsection{Control Principle Analysis of the Control System}

The control system of the heated-mandrel curing winding machine for tapered composite pole is based on the Trio motion controller. The Trio controller sends analog values of voltage to control the rotating speed of the spindle. The relationship between the voltage DAC and the rotating speed $n$ is

$$
D A C=\frac{n}{300}
$$

By closed-loop control based on the motion controller, the position of the carriage, the outrigger and the guide head is adjusted according to the PID parameters. The motion of the carriage is connected to the spindle with the electronic gear, so the carriage follows the spindle with the speed ratio of 1: $\mathrm{k}$. The value of $\mathrm{k}$ changes according to the process requirement when the carriage moves to realize the winding pattern change [11]. Meanwhile, the motion of the guide head is connected to the spindle with the electronic gear too. This speed ratio can be calculated according the width of yarn. The rotation of motor of the guide head changes the width of the yarn on the surface of the mandrel.

\subsection{Winding Pattern Design}

To obtain a stable filament winding path, this paper adopts the non-geodesic and constant winding angle to wind the power pole. The unfolding drawing of the winding pattern is shown as Figure 1 left. Since the winding angle is a constant, we can get that $\alpha 1=\alpha 2=\ldots=\alpha$. The two-dimensional section is shown as Figure 1 right, where $\mathrm{H}$ and $\mathrm{h}$ represent the radius of the big end and the small end, and L is the length of the mandrel, and $\beta$ is the taper angle of the mandrel. Take a point on the mathematical model and define it as $\mathrm{P}$. The function expression of it is

$$
F(r)=\left(1-\frac{r-h}{H-h}\right) \times L
$$

The expression of the taper angle is

$$
\tan \beta=\frac{H-h}{L}
$$

The linear velocity on the surface of mandrel is $V_{\text {sha }}$. The rotation speed of the mandrel is $V_{s}$. The speed of the carriage and the outrigger is $V_{c}$ and $V_{r}$. The speed of the guide head, which is the resultant velocity of the speed of the carriage and the outrigger, is defined as $V_{m}$. $\theta$ is the rotation angle of the spindle. So the expression of $\tan \alpha$ is

$$
\tan \alpha \approx \frac{d V_{s h a}}{d V_{m}}=\frac{r d \theta}{\sqrt{1+F^{2}(r) d r}}=\frac{1}{\sqrt{1+\left(\cot ^{2} \beta\right)}} \times \frac{V_{s}}{V_{r}}
$$


So the value of $\alpha$ is

$$
\alpha=\operatorname{arccot}\left(\frac{1}{1+\left(\cot ^{2} \beta\right)} \times \frac{V_{s}}{V_{r}}\right)
$$

According to the expressions above, the winding angle is decided by the rotation speed of the spindle and the arm.

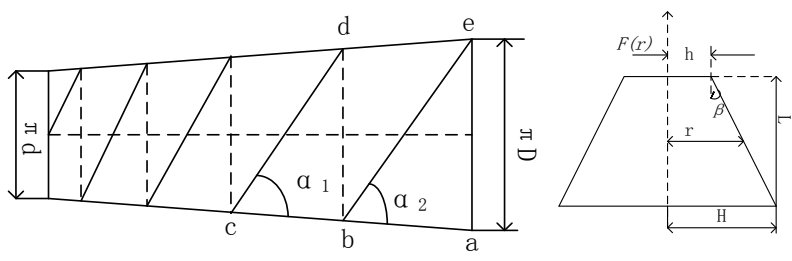

Figure 1. The Winding Pattern Design

\section{Principle of Heated-mandrel Curing and Optimization of Mandrel}

\subsection{Principle of Heated-mandrel Curing}

The structure of the mandrel of heated-mandrel curing is shown in Figure 3. The mandrel is hollow tapered cylinder. It is tapered at both ends to make the disassembly of the composite pole easier. A hollow steel pipe, called core pipe, is installed inside the mandrel coaxially, the tail of which is closed while the head is open to let in the steam. Circular holes are distributed on the surface of the core pipe as shown in Figure 2 left. The steam is pumped into the core pipe, and erupts into the cavity of the mandrel through holes to heat the surface of the mandrel in order to cure the composite wound on it. Holes are distributed at four directions to make the temperature more even and the heating faster. Steam and condensate water flow out of the mandrel from the outlet of the core pipe. Water and air come into the mandrel on the same path to cool the mandrel when needed.

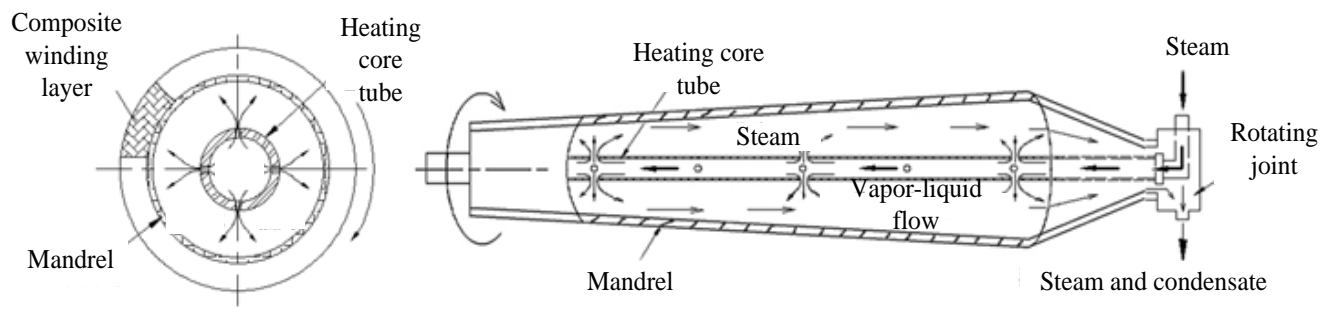

Figure 2. The Mandrel Structure of the Winding Machine

The structure of the curing system of the winding machine is show in Figure 3. The curing includes three basic processes. The first is heating process, in which the steam is introduced into the mandrel. Then, temperature keeping process begins when the temperature reaches to requirement. The temperature is controlled by adjusting the rate of flow of steam by valves installed in the entry and exit of the core pipe. The last is cooling process, in which the condensate water fills in to cool the composite, and the air comes into the pipe to clear the water. After the three processes, the composite is disassembled from the mandrel, and the heated-mandrel curing is finished. 


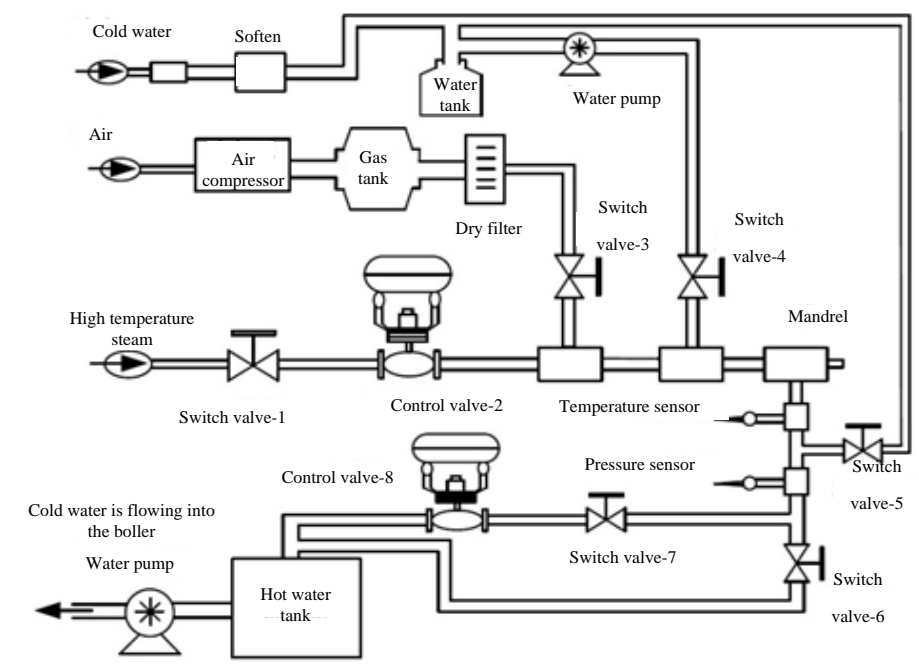

Figure 3. The Structure of the Curing System of Winding Machine

\subsection{Mandrel Structure Optimization}

The uniformity of temperature of the mandrel is essential to the curing quality of composite products. Experiments show that the temperature distribution is mainly affected by the mandrel structure. The finite element method is used to obtain the relationship between the temperature distribution and the mandrel structure. Several models of different mandrels are made, and the heating processes using these models are simulated. The results of two of the models are shown in Figure 4. From the analysis of simulation results, two problems are found. One is that the temperature of the end of the mandrel is low. Another is that the temperature near the holes is too high locally. This paper adopts the methods below to optimize the mandrel structure in order to solve the problems above. 1. Increase the amount of holes; 2. Increase the distribution density of holes in axial direction for mandrel tail; 3. Increase the diameter of mandrel tube; 4 . Increase the diameter of holes.

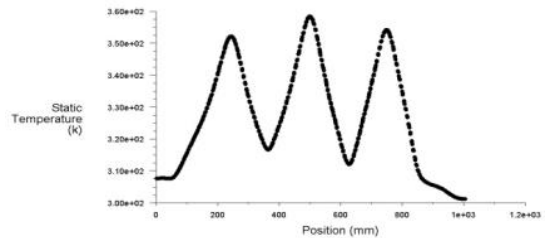

Modle 1 temperature cure at 90 s

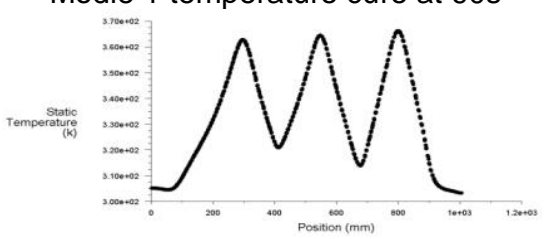

Modle 2 temperature cure at 90 s

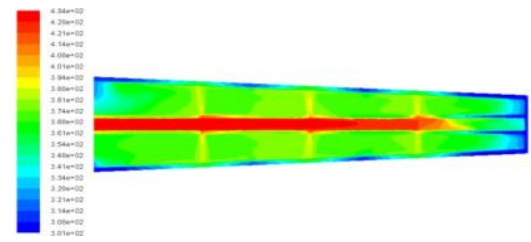

Modle 1 temperature nephogram at 90 s

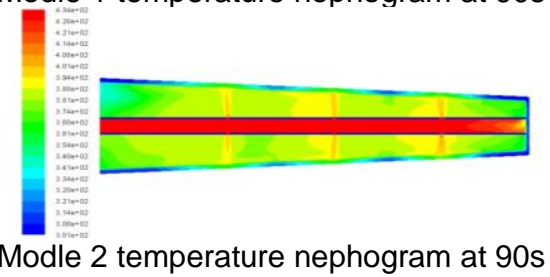

Figure 4. The Temperature Distribution of the Tapered Mandrel 
As the holes of the optimized mandrel become bigger, steam flows well at same inlet pressure, which alleviates local temperature impact. Meanwhile, the problem of the low end temperature is solved by increasing holes distribution density to increase the steam flow in tail. Tests show that the temperature difference of the optimized mandrel is $5{ }^{\circ} \mathrm{C}$ at most during the heating process, which is about $10{ }^{\circ} \mathrm{C}$ smaller than that of the traditional mandrel.

\section{Design of Winding Machine for Tapered Composite Pole}

This paper designed a four-axis tapered winding machine, which combines winding, curing, and demoulding in the integrated. Winding is accomplished by the cooperation of the rotation of the mandrel and the movement of the carriage, which are controlled by the embedded motion controller. OMRON PLC and the embedded touch screen IPC are used to complete the curing. A winding machine control system based on IPC and the embedded motion controller is designed with the structure of host and slave computer.

\subsection{Mechanical Structure Design}

The winding machine is mainly composed of four modules, which are the spindle box, the lathe bed, the winding carriage, and the winding guide head. The spindle box includes the spindle, reduction gears, and the carriage move transmission; the lathe bed is used for connecting and supporting, and the resin heating attachment and the demoulding device on it are used for avoiding the condensation of the resin in the tank and separating the pipe with the mandrel. The winding carriage mainly composed of the support, the carrier, the outrigger, and the hunting gear. The winding guide head is used for yarn-guiding. As the guide head moving with the mandrel with certain regulation, the pattern required is accomplished. The winding machine also has other auxiliary devices, such as the tensioner, the melting tank, etc. They are the integral parts for the processing of one complete product.

\subsection{Hardware Design of the Control System}

The control system of the winding machine is composed of the spindle system and the carriage system. The motion of the spindle system is that the spindle motor drives the tapered mandrel do one-way rotation. The torque provided by the servo motor increases via the reducer, and is passed to the spindle through the gearing. The carriage system is composed of the carriage, the outrigger, and the guide head. The motor drives the carriage do reciprocating motion on the horizontal direction of the spindle.

The principle of the control system is shown in Figure 5. The left half is the winding section. It is established by IPC and the Trio motion controller. The controller has contactor for the analog signal output which can be used for sending analog signal to motors to control the position and the speed of the motor. The signal is sent back by the encoder on the servo motor, and is transmitted to the motion controller after processing. In this way, the accurate control of the motor is realized. The synchronous movement control of the carriage, the outrigger, and the wire guide head is realized through the function of the electronic gear in the embedded motion controller. The right half is the curing section. It realizes the curing of the tapered pipe through the cooperation of the OMRON CJ series PLC and the embedded touch screen IPC. The curing process parameters can be set on the touch screen. The control of the temperature is realized by the controlling of the switch value of valves based on the feedback coming from the temperature sensors and the pressure sensors. 


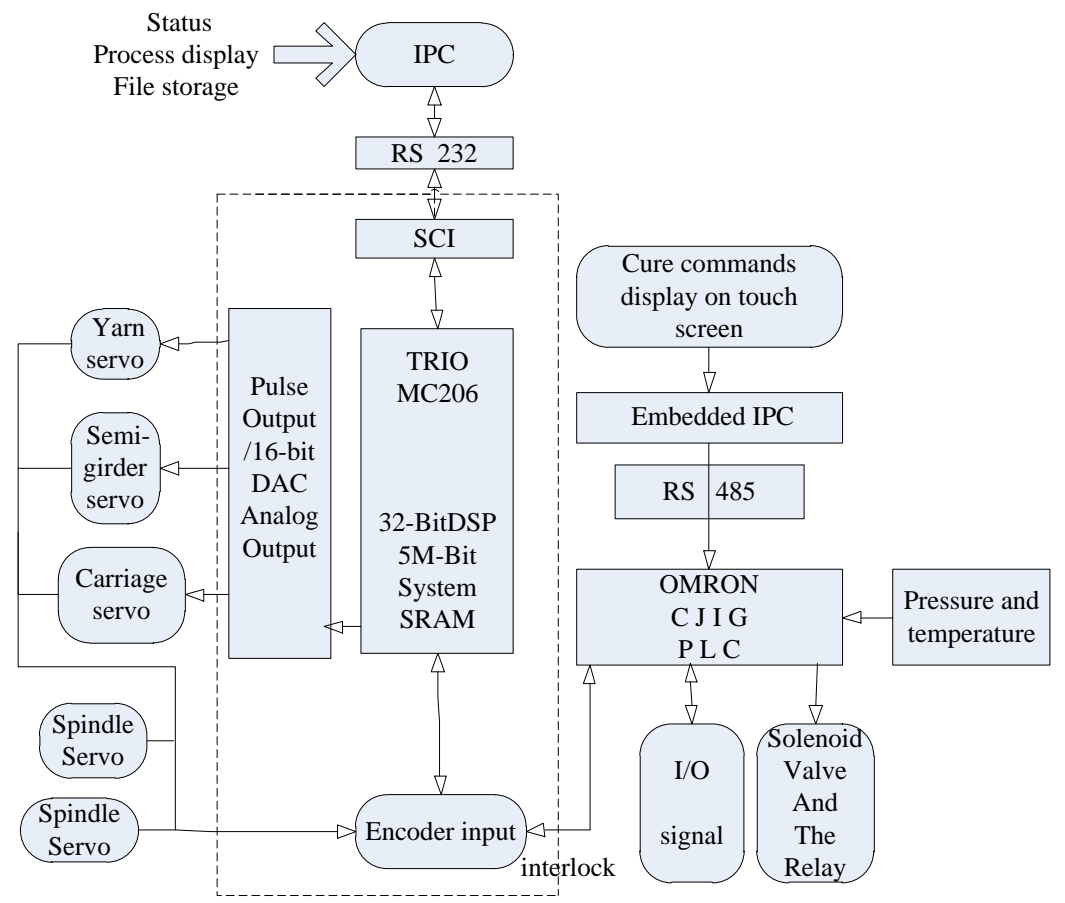

Figure 5. The Principle of the Control System

\subsection{Software Design of the Control System}

The software of the winding machine control system mainly consists of two parts. One is the host computer software, and the other is the slave computer software.

The host computer software is developed via VC++6.0 on the WINDOWS platform, which realizes serial communicating, parameter inputting and calculating, running status display, real-time data transmission, system alarm, etc. The design of the HMI is realized by using the programming language of the $\mathrm{VC}++$ and the MFC application. The MFC application can be used to realize the visualized management, and the products processing, parameter modification, program setting, function adjusting, and the status monitoring, etc. can also be realized.

The slave computer software is developed by using the Trio BASIC language provides by the Trio Motion Technology Company. The language is widely used in different types of Trio motion controller. Programs can be run in the Trio motion controller directly. In practical processing, Motion Perfect also has off-line mode, in which multi-threading operation can be realized. Thread 1 is used for completing the initialization of the system. Thread 2 is used for completing the motion control function. Programs are transmitted to the motion controller to process and record. The controller will automatically check and compile the program.

\section{Conclusions}

1. The control principle of the control system of the internal curing winding machine is analyzed in this paper. According to the process requirements of tapered composite power pole, the method of non-geodesic even winding angle stable winding is adopted. Aiming to the problem of uneven winding depth, the algorithm of winding tracks is analyzed, and the desired winding angle is calculated. 
2. The internal curing method is adopted to form the composite power pole. Aiming to the problem of uneven temperature of the mandrel, the relationship between the temperature distribution and the structure of the mandrel is analyzed according to the result of the simulation of the heating process by finite element method, and the structure of the mandrel is redesigned. Tests show that the temperature distribution becomes more even and the quality of the product is therefore improved.

3. The mechanical structure of the winding machine as well as the hardware and the software of the control system are designed. The electronic gear control method is adopted to realize the synchronized motion control of the carriage, the outrigger, and the winding guide head tracking the spindle, which improves the stability of the control system.

\section{Funding}

This work was supported by Harbin Innovative Talents of Science and Technology [grant number RC2012XK009017] and the Natural Science Foundation of Heilongiiang Province [grant number E201301].

\section{References}

[1] K. Q. Xia, High-tech Fibers and Application, vol. 30, no. 5, (2005), pp. 19-23.

[2] D. C. Hu, High-tech Fibers and Application, no. 2, (2005), pp. 49-50.

[3] M. X. Yang, Y. Chen, W. G. Li, Y. Liu, J. G. Dai and X. S. Zhao, "North China Electric Power", vol. 10, (2010), pp. 48-50.

[4] J. Z. Xu, M. Qiao, B. You and X. Y. Wang, Material Science and Technology, vol. 17, (2009), pp. 191-194.

[5] H. L. Liu, Fiber Composites, no. 1, (2011), pp. 38-40.

[6] P. W. Foumier and V. Gocevski, 2000 IEEE ESMO, (2000), pp. 358-364.

[7] S. Metiche and R Masmoudi, Journal of Composite Materials, vol. 47, no. 2, (2013), pp. 207-229.

[8] F. Amir and R. Sami, Construction and Building Materials, vol. 17, no. 6-7, (2003), pp. 507-516.

[9] R. Masmoudi, H. Mohamed and S. Metiche, Journal of Reinforced Plastics and Composites, vol. 27, no. 6, (2008), pp. 639-658.

[10] H. Z. Bi, Building Materials Industrial Information, vol. 4, no. 15, (1996). 
International Journal of $u-$ and e- Service, Science and Technology Vol.7, No.1 (2014) 